\title{
A necessary condition of potential blowup for the Navier-Stokes system in half-space
}

\author{
T. Barker $^{1}$ • G. Seregin ${ }^{1}$
}

Received: 4 December 2015 / Revised: 11 September 2016 / Published online: 31 October 2016

(C) The Author(s) 2016. This article is published with open access at Springerlink.com

Abstract Assuming that $T$ is a potential blow up time for the Navier-Stokes system in $\mathbb{R}_{+}^{3}$, we show that the norm of the velocity field in the Lorenz space $L^{3, q}$ with $q<\infty$ goes to $\infty$ as time $t$ approaches $T$.

\section{Introduction}

The question that is addressed in the paper is as follows. Let us consider the initial boundary value problem for the Navier-Stokes system in the space-time domain $Q_{+}=$ $\Omega \times] 0, \infty\left[\right.$ for vector-valued function $v=\left(v_{1}, v_{2}, v_{3}\right)=\left(v_{i}\right)$ and scalar function $q$, satisfying the equations

$$
\partial_{t} v+v \cdot \nabla v-\Delta v=-\nabla q, \quad \operatorname{div} v=0
$$

in $Q_{+}$, the boundary conditions

$$
v=0
$$

on $\partial \Omega \times[0, \infty[$, and the initial conditions

$$
v(\cdot, 0)=v_{0}(\cdot)
$$

$凶 \quad$ T. Barker

tobias.barker@seh.ox.ac.uk

G. Seregin

seregin@maths.ox.ac.uk

1 OxPDE, Mathematical Institute, University of Oxford, Oxford, UK 
in $\Omega$. It is assumed that the initial velocity field $v_{0}$ is smooth, compactly supported, and divergence free in $\Omega$, i.e., $v_{0}$ belongs to the space $C_{0,0}^{\infty}(\Omega)$, and that $\Omega$ is a domain in $\mathbb{R}^{3}$ with sufficiently smooth boundary. Here,

$$
C_{0,0}^{\infty}(\Omega):=\left\{v \in C_{0}^{\infty}(\Omega): \operatorname{div} v=0\right\}
$$

Our main aim is to study whether or not the velocity field $v$ blows up in a finite time, in other words, whether or not there exists a finite time $T>0$ such that

$$
\lim _{t \uparrow T}\|v(\cdot, t)\|_{L_{\infty}(\Omega)}=\infty
$$

There is a huge number of papers dedicated to this problem. Among them the most relevant to us are the following papers. In the first place, one should mention the classical Leray necessary conditions for $T$ to be a blowup time:

$$
\|v(\cdot, t)\|_{L_{s}(\Omega)} \geq \frac{c_{s}}{(T-t)^{\frac{s-3}{2 s}}}
$$

for any $0<t<T$, for all $s>3$, and for a positive constant $c_{s}$ depending only on $s$. Estimates (1.5) have been proven by Leray [11] for $\Omega=\mathbb{R}^{3}$ and then by Giga [5] for a wide class of domains $\Omega$ including a half space and bounded domains with sufficiently smooth boundaries. However, there is an interesting marginal case $s=3$, in which no estimate of type (1.5) is known. In papers [3,14,17], it has been shown that

$$
\limsup _{t \uparrow T}\|v(\cdot, t)\|_{L_{3}(\Omega)}=\infty
$$

for $\Omega=\mathbb{R}^{3}, \Omega=\mathbb{R}_{+}^{3}:=\left\{x=\left(x_{i}\right) \in \mathbb{R}^{3}: x_{3}>0\right\}$ and for $\Omega$ being a bounded domain with sufficiently smooth boundary.

Recent progress has been made in establishing the validity of (1.6) for other critical spaces. We refer to $X$, consisting of measurable functions acting on domains in $\mathbb{R}^{3}$, as critical if for $u \in X$ such that $u_{\lambda}(x)=\lambda u(\lambda x)$ we have $\left\|u_{\lambda}\right\|_{X}=\|u\|_{X}$. In $[15,28]$, it was shown that when $X$ is the non endpoint Lorentz space with $q$ finite $L^{3, q}\left(\mathbb{R}^{3}\right)$ condition (1.6) remains to be true. Recently, Gallagher et al. proved in [4] that (1.6) holds for $X=\dot{B}_{p, q}^{-1+\frac{3}{p}}\left(\mathbb{R}^{3}\right)$ in the framework of "strong" solutions.

Our work is motivated by the following question: what are the critical spaces $X(\Omega)$ and domains $\Omega$ for which

$$
\lim _{t \uparrow T}\|v(\cdot, t)\|_{X(\Omega)}=\infty
$$

holds true?

In [21] it is proven that this holds for $\Omega=\mathbb{R}^{3}$ and $X(\Omega)=L_{3}\left(\mathbb{R}^{3}\right)$ using the theory of local energy solutions in [10], in addition to ideas developed in $[19,20]$. The aim of the paper is to prove 
Theorem 1.1 Let $\Omega=\mathbb{R}_{+}^{3}$. Let $v: Q_{+} \rightarrow \mathbb{R}^{3}$ be a Leray-Hopf solution to (1.2)(1.3), with initial data $v_{0} \in C_{0,0}^{\infty}(\Omega)$. Suppose $3 \leqslant q<\infty$. Let $T$ be a blow-up time. Then it necessarily holds that

$$
\lim _{t \uparrow T}\|v(\cdot, t)\|_{L^{3, q}\left(\mathbb{R}_{+}^{3}\right)}=\infty
$$

Before commenting further let us define the Lorentz spaces. For a measurable function $f: \Omega \rightarrow \mathbb{R}$ define:

$$
d_{f, \Omega}(\alpha):=|\{x \in \Omega:|f(x)|>\alpha\}| .
$$

Given a measurable subset $\Omega \subset \mathbb{R}^{n}$, the Lorentz space $L^{p, q}(\Omega)$, with $\left.p \in\right] 0, \infty$, $q \in] 0, \infty$, is the set of all measurable functions $g$ on $\Omega$ such that the quasinorm $\|g\|_{L^{p, q}(\Omega)}$ is finite. Here:

$$
\begin{gathered}
\|g\|_{L^{p, q}(\Omega)}:=\left(p \int_{0}^{\infty} \alpha^{q} d_{g, \Omega}(\alpha)^{\frac{q}{p}} \frac{d \alpha}{\alpha}\right)^{\frac{1}{q}}, \\
\|g\|_{L^{p, \infty}(\Omega)}:=\sup _{\alpha>0} \alpha d_{g, \Omega}(\alpha)^{\frac{1}{p}} .
\end{gathered}
$$

It is well known that for $\left.p \in] 0, \infty\left[, q_{1} \in\right] 0, \infty\right]$ and $\left.\left.q_{2} \in\right] 0, \infty\right]$ with $q_{1} \leqslant q_{2}$ we have the following embedding $L^{p, q_{1}} \hookrightarrow L^{p, q_{2}}$ and the inclusion is known to be strict. Roughly speaking, the second index of Lorentz spaces gives information regarding nature of logarithmic bumps. For example, for any $1>\beta>0, q>3$ we have

$$
|x|^{-1}\left|\log \left(|x|^{-1}\right)\right|^{-\beta} \chi|x|<1(x) \in L^{3, q}\left(\mathbb{R}^{3}\right) \text { if and only if } q>\frac{1}{\beta} \text {. }
$$

It should be stressed that, at the time of writing, (1.7) is open for the critical norm $L^{3, \infty}(\Omega)$ that contains $|x|^{-1}$. Furthermore, uniqueness of weak Leray-Hopf solutions in the space $L_{\infty}\left(0, T ; L^{3, \infty}(\Omega)\right)$ remains open. We mention that interior regularity results, that have smallness condition on $L_{\infty}\left(L^{3, \infty}\right)$ norm, have been obtained in $[8,9,12]$, for example.

We would like to emphasise that to prove Theorem 1.1, a different approach is used to that of the whole space. Though we focus on the half space, this also provides an alternative to the proof given for the whole space in [21].

The main difficulty in attempts to prove Theorem 1.1 is as follows. The proof of this statement, for $q=3$, in the case $\Omega=\mathbb{R}^{3}$ consists of two big parts: rescaling, leading to a certain class of ancient solutions to the Navier-Stokes equations, and a Liouville type theorem for those solutions based on the backward uniqueness. The second part at least conceptually works in the case of a half space $\mathbb{R}_{+}^{3}$ as well while the first one does not. The reason is that the rescaling and the limiting procedure in the case of the whole space $\mathbb{R}^{3}$ give the special type of the so-called local energy 
ancient solutions to the Navier-Stokes that coincide with Lemarie-Rieusset solutions to the Cauchy problem for the Navier-Stokes equations on some finite time interval. Those solutions have been introduced by Lemarie-Rieusset [10], see also for some definitions in [7]. Unfortunately, an analogue of Lemarie-Rieusset solutions for a half space is not known yet. In fact, this is an interesting open problem. In this paper, we are able to work without Lemarie-Rieusset type solutions in half space to get a local energy ancient solution to which a Liouville type theorem based on backward uniqueness is applicable. In comparison to [21], our approach doesn't require a notion of local energy solution, since we get all desired properties for the ancient solution directly from a priori estimates associated to the blow up procedure. Those ideas can be used for the construction of a global in time solution,

for non energy initial data that is contained in the sum of certain Lesbesgue spaces, that applies in $\mathbb{R}^{3}$ and is extendible to other unbounded domains with boundaries. Further developments in this direction will be published elsewhere. ${ }^{1}$

\section{Preliminaries}

In this section, we will introduce notation that will be repeatedly used throughout the rest of the paper. For spatial domains and space time domains, we will make use of the following notation:

$$
\begin{aligned}
B\left(x_{0}, R\right) & =\left\{x \in \mathbb{R}^{3}:\left|x-x_{0}\right|<R\right\} \\
B(\theta) & =B(0, \theta), \quad B=B(1), \\
Q\left(z_{0}, R\right) & \left.=B\left(x_{0}, R\right) \times\right] t_{0}-R^{2}, t_{0}\left[, \quad z_{0}=\left(x_{0}, t_{0}\right),\right. \\
Q(\theta) & \left.=Q(0, \theta), Q=Q(1), \quad Q_{-T, 0}:=\mathbb{R}^{3} \times\right]-T, 0[, \\
B^{+}\left(x_{0}, R\right) & =\left\{x \in B\left(x_{0}, R\right): x=\left(x^{\prime}, x_{3}\right), x_{3}>x_{03}\right\}, \\
B^{+}(\theta) & =B^{+}(0, \theta), \quad B^{+}=B^{+}(1), \\
Q^{+}\left(z_{0}, R\right) & \left.=B^{+}\left(x_{0}, R\right) \times\right] t_{0}-R^{2}, t_{0}[, \\
Q^{+}(\theta) & \left.=Q^{+}(0, \theta), \quad Q^{+}=Q^{+}(1), Q_{-T, 0}^{+}:=\mathbb{R}_{+}^{3} \times\right]-T, 0[, \\
\mathbb{R}_{+\delta}^{3} & =\left\{\left(x^{\prime}, x_{3}\right) \in \mathbb{R}_{+}^{3}: x_{3} \geq \delta\right\} .
\end{aligned}
$$

For $\Omega \subset \mathbb{R}^{3}$, mean values of integrable functions are denoted as follows

$$
[p]_{\Omega}=\frac{1}{|\Omega|} \int_{\Omega} p(x) d x .
$$

For, $\Omega \subset \mathbb{R}^{3}$, the space $\left[C_{0,0}^{\infty}(\Omega)\right]^{L_{s}(\Omega)}$ is defined to be the closure of

$$
C_{0,0}^{\infty}(\Omega):=\left\{u \in C_{0}^{\infty}(\Omega): \operatorname{div} u=0\right\}
$$

\footnotetext{
1 After submission of this manuscript, the second author and Šverák have carried this out for data in $L_{3}$. See [22].
} 
with respect to the $L_{S}(\Omega)$ norm. If $X$ is a Banach space with norm $\|\cdot\|_{X}$, then $L_{s}(a, b ; X), a<b$, will denote the usual Banach space of strongly measurable $X$ valued functions $f(t)$ on $] a, b$ [ such that the norm

$$
\|f\|_{L_{s}(a, b ; X)}:=\left(\int_{a}^{b}\|f(t)\|_{X}^{s} d t\right)^{\frac{1}{s}}<+\infty
$$

for $s \in[1, \infty[$, and with the usual modification if $s=\infty$. With this notation, we will define

$$
\begin{aligned}
& L_{s, l}\left(Q_{-T, 0}\right):=L_{l}\left(-T, 0 ; L_{s}\left(\mathbb{R}^{3}\right)\right) \\
& L_{s, l}\left(Q_{-T, 0}^{+}\right):=L_{l}\left(-T, 0 ; L_{s}\left(\mathbb{R}_{+}^{3}\right)\right)
\end{aligned}
$$

We define the following Sobolev spaces with the mixed norm:

$$
\begin{aligned}
W_{m, n}^{1,0}\left(Q_{-T, 0}^{+}\right)= & \left\{v \in L_{m, n}\left(Q_{-T, 0}^{+}\right):\|v\|_{L_{m, n}\left(Q_{-T, 0}^{+}\right)}\right. \\
& \left.+\|\nabla v\|_{L_{m, n}\left(Q_{-T, 0}^{+}\right)}<\infty\right\}, \\
W_{m, n}^{2,1}\left(Q_{-T, 0}^{+}\right)= & \left\{v \in L_{m, n}\left(Q_{-T, 0}^{+}\right):\|v\|_{L_{m, n}\left(Q_{-T, 0}^{+}\right)}\right. \\
& \left.+\left\|\nabla^{2} v\right\|_{L_{m, n}\left(Q_{-T, 0}^{+}\right)}+\left\|\partial_{t} v\right\|_{L_{m, n}\left(Q_{-T, 0}^{+}\right)}<\infty\right\} .
\end{aligned}
$$

The analogues definitions for the above Sobolev spaces hold when the space-time domain is replaced by $Q_{-T, 0}$.

\section{A priori estimates}

First we begin by stating and proving a simple (but important) fact about Lorentz spaces concerning a decomposition. This will be formulated as a Lemma. Analogous statement is Lemma II.I proven by Calderon [2].

Lemma 3.1 Take $1<t<r<s \leqslant \infty$, and suppose that $g \in L^{r, \infty}(\Omega)$. For any $N>0$, we let $g_{N_{-}}:=g \chi_{|g| \leqslant N}$ and $g_{N_{+}}:=g-g_{N_{-}}$. Then

$$
\left\|g_{N_{-}}\right\|_{L_{s}(\Omega)}^{s} \leqslant \frac{s}{s-r} N^{s-r}\|g\|_{L^{r, \infty}(\Omega)}^{r}
$$

if $s<\infty$ and $\left\|g_{N_{-}}\right\|_{L_{\infty}(\Omega)} \leqslant N$, and

$$
\left\|g_{N_{+}}\right\|_{L_{t}(\Omega)}^{t} \leqslant \frac{r}{r-t} N^{t-r}\|g\|_{L^{r, \infty}(\Omega)}^{r} .
$$

Moreover for $\Omega=\mathbb{R}^{3}$ or $\mathbb{R}_{+}^{3}$, if $g \in L^{r, p}(\Omega)$ with $1 \leqslant p \leq \infty$ and $\operatorname{div} g=0$ in weak sense (also $g_{3}\left(x^{\prime}, 0\right)=0$ for half space in weak sense), then $g=g_{1}+g_{2}$ where 
$g_{1} \in\left[C_{0,0}^{\infty}(\Omega)\right]^{L_{s}(\Omega)}$ with

$$
\left\|g_{1}\right\|_{L_{s}(\Omega)} \leqslant C\left(s, r, p,\|g\|_{L^{r, p}(\Omega)}\right)
$$

and $g_{2} \in\left[C_{0,0}^{\infty}(\Omega)\right]^{L_{t}(\Omega)}$ with

$$
\left\|g_{2}\right\|_{L_{t}(\Omega)} \leqslant C\left(r, t, p,\|g\|_{L^{r, p}(\Omega)}\right) .
$$

Proof Proof of decomposition (3.1), (3.2) can be found in [13].

Given $g$, satisfying assumptions of the lemma, we can find $g_{1_{-}}$and $g_{1_{+}}$. We then can use the Helmoltz-Weyl decomposition $g_{1_{-}}=g_{1}+\nabla q_{1}$ where $g_{1}$ belongs to the required space with the estimate $\left\|g_{1}\right\|_{L_{s}(\Omega)} \leq c\left\|g_{1_{-}}\right\|_{L_{S}(\Omega)},\left\|\nabla q_{1}\right\|_{L_{s}(\Omega)} \leq$ $c\left\|g_{1_{-}}\right\|_{L_{s}(\Omega)}$, and

$$
\int_{\Omega} \nabla q_{1} \cdot \nabla \varphi d x=\int_{\Omega} g_{1_{-}} \cdot \nabla \varphi d x, \quad \forall \varphi \in C_{0}^{\infty}\left(\mathbb{R}^{3}\right) .
$$

The same is true for the second counterpart. So, we have

$$
\int_{\Omega} \nabla\left(q_{1}+q_{2}\right) \cdot \nabla \varphi d x=0 \quad \forall \varphi \in C_{0}^{\infty}\left(\mathbb{R}^{3}\right) .
$$

Using properties of harmonic functions and the above global integrability of $\nabla q_{1}$ and $\nabla q_{2}$, we conclude that $\nabla\left(q_{1}+q_{2}\right)=0$. From this, from estimates (3.1), (3.2), and embedding $L^{r, p}(\Omega)$ into $L^{r, \infty}(\Omega)$, we derive the required estimates (3.3) and (3.4).

Let us consider a sufficiently smooth solution $u$ and $p$ to the Navier-Stokes system in the space-time strip $\left.Q_{-2,0}^{+}=\mathbb{R}_{+}^{3} \times\right]-2,0[$ to the following initial boundary value problem:

$$
\partial_{t} u+\operatorname{div} u \otimes u-\Delta u=-\nabla p, \quad \operatorname{div} u=0
$$

in $Q_{-2,0}^{+}$,

$$
u\left(x^{\prime}, 0, t\right)=0
$$

for $\left(x^{\prime}, t\right) \in \mathbb{R}^{2} \times[-2,0]$,

$$
u(\cdot,-2)=u_{0}(\cdot) \in L^{3, q}\left(\mathbb{R}_{+}^{3}\right) .
$$

Suppose that

$$
\left\|u_{0}\right\|_{L^{3, q}\left(\mathbb{R}_{+}^{3}\right)}=M<\infty .
$$

The key observation for Lorentz spaces is as follows. From Lemma 3.1 (along with fact embedding into weak $L_{3}$ ) we may decompose: 


$$
u_{0}:=u_{0}^{1}+u_{0}^{2}
$$

where

$$
\begin{gathered}
u_{0}^{1} \in\left[C_{0,0}^{\infty}\left(\mathbb{R}_{+}^{3}\right)\right]^{L_{\frac{10}{3}}\left(\mathbb{R}_{+}^{3}\right)}, u_{0}^{2} \in\left[C_{0,0}^{\infty}\left(\mathbb{R}_{+}^{3}\right)\right]^{L_{2}\left(\mathbb{R}_{+}^{3}\right)} \\
\left\|u_{0}^{1}\right\|_{L_{\frac{10}{3}}\left(\mathbb{R}_{+}^{3}\right)}+\left\|u_{0}^{2}\right\|_{L_{2}\left(\mathbb{R}_{+}^{3}\right)} \leqslant c(M, q) .
\end{gathered}
$$

We then decompose $u=v^{1}+v^{2}$ where $v^{1}$ and $p^{1}$ solve the linear problem

$$
\partial_{t} v^{1}-\Delta v^{1}=-\nabla p^{1}, \quad \operatorname{div} v^{1}=0
$$

in $Q_{-2,0}^{+}$,

$$
v^{1}\left(x^{\prime}, 0, t\right)=0
$$

for $\left(x^{\prime}, t\right) \in \mathbb{R}^{2} \times[-2,0]$,

$$
v^{1}(\cdot,-2)=u_{0}^{1}(\cdot,-2) \in L_{\frac{10}{3}}\left(\mathbb{R}_{+}^{3}\right) .
$$

Below we give some relevant estimates for the linear problem. We note that the cases $k=0,1$ and $\frac{10}{3} \leqslant l<\infty$ follow from [27, Theorem 3.1]. The case $l=\infty$ can be obtained directly by using Solonnikov estimates for the Green function in half-space, see [26]. The relevant estimates are $\left(\frac{10}{3} \leqslant l \leqslant \infty, k=0,1, \ldots\right)$ :

$$
\left\|\nabla^{k} v^{1}(\cdot, t)\right\|_{L_{l}\left(\mathbb{R}_{+}^{3}\right)} \leqslant \frac{c(M)}{(t+2)^{\frac{k}{2}+\frac{3}{2}\left(\frac{3}{10}-\frac{1}{l}\right)}} .
$$

Thus

$$
\left\|v^{1}(\cdot, t)\right\|_{L_{5}\left(\mathbb{R}_{+}^{3}\right)} \leqslant \frac{c(M)}{(t+2)^{\frac{3}{20}}}, \quad\left\|v^{1}(\cdot, t)\right\|_{L_{4}\left(\mathbb{R}_{+}^{3}\right)} \leqslant \frac{c(M)}{(t+2)^{\frac{3}{40}}} .
$$

It is then seen that:

$$
\left\|v^{1}\right\|_{L_{5}\left(Q_{-2,0}^{+}\right)}+\left\|v^{1}\right\|_{L_{4}\left(Q_{-2,0}^{+}\right)}+\sup _{t \in]-2,0[}\left\|v^{1}(\cdot, t)\right\|_{L_{\frac{10}{3}}\left(\mathbb{R}_{+}^{3}\right)} \leqslant c(M)
$$

The second counterpart of $u$ satisfies the non-linear system

$$
\partial_{t} v^{2}+\operatorname{div} u \otimes u-\Delta v^{2}=-\nabla p^{2}, \quad \operatorname{div} v^{2}=0
$$


in $Q_{-2,0}^{+}$, the boundary conditions

$$
v^{2}\left(x^{\prime}, 0, t\right)=0
$$

for $\left(x^{\prime}, t\right) \in \mathbb{R}^{2} \times[-2,0]$, and the initial conditions

$$
v^{2}(\cdot,-2)=u_{0}^{2}
$$

in $\mathbb{R}_{+}^{3}$.

The standard energy approach to the second system gives

$$
\partial_{t}\left\|v^{2}(\cdot, t)\right\|_{L_{2}\left(\mathbb{R}_{+}^{3}\right)}^{2}+2\left\|\nabla v^{2}(\cdot, t)\right\|_{L_{2}\left(\mathbb{R}_{+}^{3}\right)}^{2}=2 \int_{\mathbb{R}_{+}^{3}} u \otimes u: \nabla v^{2} d x d s=I_{1}+I_{2},
$$

where

$$
I_{1}=2 \int_{\mathbb{R}_{+}^{3}} v^{1} \otimes v^{1}: \nabla v^{2} d x, \quad I_{2}=2 \int_{\mathbb{R}_{+}^{3}} v^{1} \otimes v^{2}: \nabla v^{2} d x
$$

Next, let us consequently evaluate terms on the right hand side of the energy identity. For the first term, we have

$$
\left|I_{1}\right| \leq c\left\|v^{1}(\cdot, t)\right\|_{L_{4}\left(\mathbb{R}_{+}^{3}\right)}^{2}\left\|\nabla v^{2}(\cdot, t)\right\|_{L_{2}\left(\mathbb{R}_{+}^{3}\right)} .
$$

The second term can be treated as follows:

$$
\begin{aligned}
\left|I_{2}\right| & \leq c\left\|v^{1}(\cdot, t) \otimes v^{2}(\cdot, t)\right\|_{L_{2}\left(\mathbb{R}_{+}^{3}\right)}\left\|\nabla v^{2}(\cdot, t)\right\|_{L_{2}\left(\mathbb{R}_{+}^{3}\right)} \\
& \leq c\left\|v^{1}(\cdot, t)\right\|_{L_{5}\left(\mathbb{R}_{+}^{3}\right)}\left\|v^{2}(\cdot, t)\right\|_{L_{\frac{10}{3}}\left(\mathbb{R}_{+}^{3}\right)}\left\|\nabla v^{2}(\cdot, t)\right\|_{L_{2}\left(\mathbb{R}_{+}^{3}\right)} .
\end{aligned}
$$

Applying the known multiplicative inequality to the second factor in the right hand side of the latter bound, we find

$$
\left|I_{2}\right| \leq c\left\|v^{1}(\cdot, t)\right\|_{L_{5}\left(\mathbb{R}_{+}^{3}\right)}\left\|v^{2}(\cdot, t)\right\|_{L_{2}\left(\mathbb{R}_{+}^{3}\right)}^{\frac{2}{5}}\left\|\nabla v^{2}(\cdot, t)\right\|_{L_{2}\left(\mathbb{R}_{+}^{3}\right)}^{\frac{8}{5}} .
$$

\section{Letting}

$$
y(t):=\left\|v^{2}(\cdot, t)\right\|_{L_{2}\left(\mathbb{R}_{+}^{3}\right)}^{2}
$$

and using the Young inequality, we find

$$
y^{\prime}(t)+\left\|\nabla v^{2}(\cdot, t)\right\|_{L_{2}\left(\mathbb{R}_{+}^{3}\right)}^{2} \leq c\left\|v^{1}(\cdot, t)\right\|_{L_{5}\left(\mathbb{R}_{+}^{3}\right)}^{5} y(t)+c\left\|v^{1}(\cdot, t)\right\|_{L_{4}\left(\mathbb{R}_{+}^{3}\right)}^{4} .
$$


Next, elementary arguments lead to the inequality

$$
\begin{aligned}
& \left(y(t) \exp \left(-c \int_{-2}^{t}\left\|v^{1}(\cdot, s)\right\|_{L_{5}\left(\mathbb{R}_{+}^{3}\right)}^{5} d s\right)\right)^{\prime} \\
& \leq c \exp \left(-c \int_{-2}^{t}\left\|v^{1}(\cdot, s)\right\|_{L_{5}\left(\mathbb{R}_{+}^{3}\right)}^{5} d s\right)\left\|v^{1}(\cdot, t)\right\|_{L_{4}\left(\mathbb{R}_{+}^{3}\right)}^{4} .
\end{aligned}
$$

So,

$$
\begin{aligned}
y(t) \leq & c \int_{-2}^{t} \exp \left(c \int_{\tau}^{t}\left\|v^{1}(\cdot, s)\right\|_{L_{5}\left(\mathbb{R}_{+}^{3}\right)}^{5} d s\right)\left\|v^{1}(\cdot, \tau)\right\|_{L_{4}\left(\mathbb{R}_{+}^{3}\right)}^{4} d \tau \\
& +\left\|u_{0}^{2}\right\|_{L_{2}\left(\mathbb{R}_{+}^{3}\right)}^{2} \exp \left(c \int_{-2}^{t}\left\|v^{1}(\cdot, s)\right\|_{L_{5}\left(\mathbb{R}_{+}^{3}\right)}^{5} d s\right)
\end{aligned}
$$

Using (3.9) and (3.11), it is easily seen that

$$
\sup _{-2<t<0} y(t) \leqslant C(M), \quad\left\|\nabla v^{2}\right\|_{L_{2}\left(Q_{-2,0}^{+}\right)} \leqslant C(M) .
$$

From these estimates and from the multiplicative inequality, one can deduce that

$$
\left\|v^{2}\right\|_{L_{s}\left(Q_{-2,0}^{+}\right)} \leq C(s, M)
$$

with any $s \in\left[2, \frac{10}{3}\right]$. Moreover,

$$
\|u\|_{L_{\frac{10}{3}}}\left(Q_{-2,0}^{+}\right) \leq C(M) .
$$

Let

$$
f=u \cdot \nabla u \text {. }
$$

Using (3.10) and (3.12), one can decompose

$$
f=f_{1}+f_{2}+f_{3} .
$$

Here,

$$
\begin{aligned}
& f_{1}:=v^{2} \cdot \nabla v^{2} \\
& f_{2}:=v^{1} \cdot \nabla v^{1}+v^{2} \cdot \nabla v^{1} \\
& f_{3}:=v^{1} \cdot \nabla v^{2}
\end{aligned}
$$


Using (3.12) and multiplicative inequalities, one can readily verify that

$$
\left\|f_{1}\right\|_{L_{s, l}\left(Q_{-2,0}^{+}\right)} \leqslant C(M, s, l)
$$

provided that

$$
\frac{3}{s}+\frac{2}{l}=4
$$

Using (3.10) we see that

$$
\left\|f_{2}\right\|_{L_{\frac{10}{3}}\left(Q_{-\frac{7}{4}, 0}^{+}\right)} \leqslant C(M)
$$

Finally, using (3.12) and (3.10) we see that

$$
\left\|f_{3}\right\|_{L_{2}\left(Q_{-\frac{7}{4}, 0}^{+}\right)} \leqslant C(M)
$$

Putting everything together we have

$$
\left\|f_{1}\right\|_{L_{\frac{9}{8}, \frac{3}{2}}\left(Q_{-\frac{7}{4}, 0}^{+}\right)}+\left\|f_{2}\right\|_{L_{\frac{10}{3}}\left(Q_{-\frac{7}{4}, 0}^{+}\right)}+\left\|f_{3}\right\|_{L_{2}\left(Q_{-\frac{7}{4}, 0}^{+}\right)}+\|u\|_{L_{\frac{10}{3}}\left(Q_{-\frac{7}{4}, 0}^{+}\right)} \leqslant C(M) .
$$

Let us fix a smooth cut-off function $\chi(t)$ so that $\chi(t)=1$ if $-3 / 2<t<1$ and $\chi(t)=0$ if $-2<t<-7 / 4$. Then, we may split $\chi u$ and $\chi p$ in the following way:

$$
\chi u=u^{1}+u^{2}+u^{3}
$$

and

$$
\chi p=p^{1}+p^{2}+p^{3}
$$

so that,

$$
\partial_{t} u^{i}-\Delta u^{i}+\nabla p^{i}=g_{i}, \quad \operatorname{div} u^{i}=0
$$

in $Q_{-2,0}^{+}$,

$$
u^{i}\left(x^{\prime}, 0, t\right)=0
$$

for all $\left(x^{\prime}, t\right) \in \mathbb{R}^{2} \times[-2,0]$ and

$$
u^{i}(x,-2)=0
$$


for $x \in \mathbb{R}_{+}^{2}$, where

$$
g_{i}(x, t)=-\chi(t) f_{i}(x, t)+\delta_{i 2} \chi^{\prime}(t) u(x, t) .
$$

Our main tool here is the coercive estimates of the linear theory for the Stokes system developed in [25] when space and time exponents are the same and subsequently in $[6,24]$ for unequal space time exponents. In particular, using (3.14) and (3.18)-(3.20) it follows from that

$$
\begin{aligned}
& \left\|\partial_{t} u^{1}\right\|_{L_{\frac{9}{8}, \frac{3}{2}}\left(Q_{-2,0}^{+}\right)}+\left\|\nabla^{2} u^{1}\right\|_{L_{\frac{9}{8}, \frac{3}{2}}\left(Q_{-2,0}^{+}\right)} \\
& +\left\|\nabla p^{1}\right\|_{L_{\frac{9}{8}, \frac{3}{2}}\left(Q_{-2,0}^{+}\right)} \leq c\left\|g_{1}\right\|_{L_{\frac{9}{8}, \frac{3}{2}}\left(Q_{-2,0}^{+}\right)} \leq C_{1}(M) \\
& \left\|\partial_{t} u^{2}\right\|_{L_{\frac{10}{3}}\left(Q_{-2,0}^{+}\right)}+\left\|\nabla^{2} u^{2}\right\|_{L_{\frac{10}{3}}}\left(Q_{-2,0}^{+}\right) \\
& +\left\|\nabla p^{2}\right\|_{L_{\frac{10}{3}}\left(Q_{-2,0}^{+}\right)} \leq c\left\|g_{2}\right\|_{L_{\frac{10}{3}}\left(Q_{-2,0}^{+}\right)} \leq C_{2}(M)
\end{aligned}
$$

and

$\left\|\partial_{t} u^{3}\right\|_{L_{2}\left(Q_{-2,0}^{+}\right)}+\left\|\nabla^{2} u^{3}\right\|_{L_{2}\left(Q_{-2,0}^{+}\right)}+\left\|\nabla p^{3}\right\|_{L_{2}\left(Q_{-2,0}^{+}\right)} \leq c\left\|g_{3}\right\|_{L_{2}\left(Q_{-2,0}^{+}\right)} \leq C_{3}(M)$

In what follows, we are going to use the following Poincare type inequalities:

$$
\begin{aligned}
& \int_{-3 / 2}^{0} \int_{B\left(x_{0}, R\right)}\left|p^{1}-\left[p^{1}\right]_{B\left(x_{0}, R\right)}\right|^{\frac{3}{2}} d x d t \leq c R^{\frac{1}{2}} \int_{-3 / 2}^{0}\left(\int_{B\left(x_{0}, R\right)}\left|\nabla p^{1}\right|^{\frac{9}{8}} d x\right)^{\frac{4}{3}} d t \\
& \int_{-3 / 2}^{0} \int_{B\left(x_{0}, R\right)} \mid p^{2}-\left[p^{2}\right]_{B\left(x_{0}, R\right)^{\frac{3}{2}}} d x d t \leq c R^{\frac{63}{20}} \int_{-3 / 2}^{0}\left(\int_{B\left(x_{0}, R\right)}\left|\nabla p^{2}\right|^{\frac{10}{3}} d x\right)^{\frac{9}{20}} d t \\
& \int_{-3 / 2}^{0} \int_{B\left(x_{0}, R\right)}\left|p^{3}-\left[p^{3}\right]_{B\left(x_{0}, R\right)}\right|^{\frac{3}{2}} d x d t \leq c R^{\frac{9}{4}} \int_{-3 / 2}^{0}\left(\int_{B\left(x_{0}, R\right)}\left|\nabla p^{3}\right|^{2} d x\right)^{\frac{3}{4}} d t .
\end{aligned}
$$

All the formulae are valid provided $B\left(x_{0}, R\right) \subset \mathbb{R}_{+}^{3}$. They are also valid if we replace $B\left(x_{0}, R\right)$ with semi-balls $B^{+}\left(x_{0}, R\right)$ assuming that $x_{03}=0$. 


\section{Passage to the limit}

Suppose that we have a sequence of sufficiently smooth functions $u^{(n)}$ and $p^{(n)}$ defined in the domain $\left.Q_{-2,0}^{+}=\mathbb{R}_{+}^{3} \times\right]-2,0[$ that are solutions to the following initial boundary value problem:

$$
\partial_{t} u^{(n)}+\operatorname{div} u^{(n)} \otimes u^{(n)}-\Delta u^{(n)}=-\nabla p^{(n)}, \quad \operatorname{div} u^{(n)}=0
$$

in $Q_{-2,0}^{+}$,

$$
u^{(n)}\left(x^{\prime}, 0, t\right)=0
$$

for $\left(x^{\prime}, t\right) \in \mathbb{R}^{2} \times[-2,0]$,

$$
u^{(n)}(\cdot,-2)=u_{0}^{(n)}(\cdot) \in L^{3, q}\left(\mathbb{R}_{+}^{3}\right) .
$$

It is supposed also that

$$
u_{0}^{(n)} \rightarrow u_{0}
$$

in $L^{3, q}\left(\mathbb{R}_{+}^{3}\right)$.

We let

$$
M:=\sup _{n}\left\|u_{0}^{(n)}\right\|_{L^{3, q}\left(\mathbb{R}_{+}^{3}\right)}<\infty .
$$

Proposition 4.1 There exist subsequences still denoted in the same way with the following properties:

$$
u^{(n)} \rightarrow u
$$

in $L_{\frac{10}{3}}\left(Q_{-2,0}^{+}\right)$,

$$
\nabla u^{(n)} \rightarrow \nabla u
$$

in $L_{2}\left(B^{+}(R) \times\right]-2+\delta$, 0[) for any $R>0$ and any $0<\delta<2$,

$$
u^{(n)} \rightarrow u
$$

in $L_{3}\left(B^{+}(R) \times\right]-3 / 2$, 0[) for any $R>0$ and

$$
p^{(n)} \rightarrow p
$$

in $L_{\frac{3}{2}}\left(B^{+}(R) \times\right]-3 / 2,0[)$ for any $R>0$.

Functions $u$ and $p$ satisfy (3.5) in $Q_{-3 / 2,0}^{+}$and (3.6) for $\left.\left(x^{\prime}, t\right) \in \mathbb{R}^{2} \times\right]-3 / 2,0[$. 
For the pressure $p$, the following global estimates are valid:

$$
p=\sum_{i=1}^{3} p^{i}
$$

with the estimates

$$
\left\|\nabla p^{1}\right\|_{L_{\frac{9}{8}, \frac{3}{2}}\left(Q_{-3 / 2,0}^{+}\right)}+\left\|\nabla p^{2}\right\|_{L_{\frac{10}{3}}\left(Q_{-3 / 2,0}^{+}\right)}++\left\|\nabla p^{3}\right\|_{L_{2}\left(Q_{-3 / 2,0}^{+}\right)} \cdot<\infty
$$

Moreover, for any $R>0$, the limits pair $u$ and $p$ satisfies the local energy inequality

$$
\begin{aligned}
& \quad \int_{B\left(x_{0}, R\right) \cap \mathbb{R}_{+}^{3}} \varphi^{2}(x, t)|u(x, t)|^{2} d x+2 \int_{t_{0}-R^{2}}^{t} \int_{B\left(x_{0}, R\right) \cap \mathbb{R}_{+}^{3}} \varphi^{2}|\nabla u|^{2} d x d t \\
& \leq \int_{t_{0}-R^{2}}^{t} \int_{\left.B\left(x_{0}, R\right)\right) \cap \mathbb{R}_{+}^{3}}|u|^{2}\left(\partial_{t} \varphi^{2}+\Delta \varphi^{2}\right)+u \cdot \nabla \varphi^{2}\left(|u|^{2}+2 p\right) d x d s
\end{aligned}
$$

for all $-3 / 2<t_{0}-R^{2}<t \leq t_{0} \leq 0$, for all $x_{0} \in \mathbb{R}^{3}$, and for all $\varphi \in$ $C_{0}^{\infty}\left(B\left(x_{0}, R\right) \times\right] t_{0}-R^{2}, t_{0}+R^{2}[)$.

Proof Obviously, we may assume, without loss of generality, that (4.4) follows from (3.14) . Moreover, the limit function obeys the estimate

$$
\|u\|_{L_{10 / 3}\left(Q_{-2,0}^{+}\right)}<\infty
$$

Obviously, (3.10) and (3.12) imply (4.5). From (3.22)-(3.24), we may split $u^{(n)}=$ $u^{(n), 1}+u^{(n), 2}+u^{(n), 3}$ such that for $(x, t) \in Q_{-\frac{3}{2}, 0}^{+}$we have the following uniform bounds:

$$
\sup _{n}\left(\left\|u^{(n), 1}\right\|_{W_{\frac{9}{8}, \frac{3}{2}}^{2,1}\left(Q_{-\frac{3}{2}, 0}^{+}\right)}+\left\|u^{(n), 2}\right\|_{W_{\frac{10}{3}}^{2,1}\left(Q_{-\frac{3}{2}, 0}^{+}\right)}+\left\|u^{(n), 3}\right\|_{W_{2}^{2,1}\left(Q_{-\frac{3}{2}, 0}^{+}\right)}\right) \leq C(M) .
$$

By known compact embeddings we infer, that there exists a subsequence (denoted here by the original sequence) such that the following holds for any $a>0$ :

$$
\begin{gathered}
u^{(n), 1} \rightarrow u^{1} \quad \text { in } \quad C\left([-3 / 2,0] ; L_{\frac{9}{8}}\left(B^{+}(a)\right)\right), \\
u^{(n), 2} \rightarrow u^{2} \quad \text { in } \quad C\left([-3 / 2,0] ; L_{\frac{10}{3}}\left(B^{+}(a)\right)\right), \\
u^{(n), 3} \rightarrow u^{3} \quad \text { in } \quad C\left([-3 / 2,0] ; L_{2}\left(B^{+}(a)\right)\right) .
\end{gathered}
$$


Using the above, along with the fact $u^{(n)}$ is bounded in $L_{\frac{10}{3}}\left(Q_{-2,0}^{+}\right)$, we can deduce (4.6).

Now, let us treat the pressure $p^{(n)}$, using the decomposition of the previous section

$$
p^{(n)}=\sum_{i=1}^{3} p^{(n), i} .
$$

Then, using (3.22)-(3.24) and (3.25)-(3.27), we can justify (4.7) and (4.8).

Since functions $u^{(n)}$ and $p^{(n)}$ satisfy the local energy inequality, i.e.,

$$
\begin{aligned}
& \left.\int_{B\left(x_{0}, R\right) \cap \mathbb{R}_{+}^{3}}\left|\varphi^{2}(x, t)\right| u^{(n)}(x, t)\right|^{2} d x+2 \int_{t_{0}-R^{2}}^{t} \int_{B\left(x_{0}, R\right) \cap \mathbb{R}_{+}^{3}} \varphi^{2}\left|\nabla u^{(n)}\right|^{2} d x d t \\
& \leq \int_{t_{0}-R^{2} B\left(x_{0}, R\right) \cap \mathbb{R}_{+}^{3}}^{t}\left|u^{(n)}\right|^{2}\left(\partial_{t} \varphi^{2}+\Delta \varphi^{2}\right)+u^{(n)} \cdot \nabla \varphi^{2}\left(\left|u^{(n)}\right|^{2}+2 p^{(n)}\right) d x d s
\end{aligned}
$$

for all $-2<t_{0}-R^{2}<t \leq t_{0} \leq 0$, for all $x_{0} \in \mathbb{R}^{3}$, and for all $\varphi \in C_{0}^{\infty}\left(B\left(x_{0}, R\right) \times\right] t_{0}-$ $R^{2}, t_{0}+R^{2}$ [), we can find (4.9) by passing to the limits and taking into account (4.6) and (4.7).

Proposition 4.2 Let $u$ and $p$ be a limit function from Proposition 4.1. There exists a number $R_{1}>0$ such that

$$
|u(x, t)| \leq c
$$

for all $\left.(x, t) \in\left(\mathbb{R}_{+}^{3} \backslash B^{+}\left(R_{1}\right)\right) \times\right]-5 / 4,0[$ and for some universal constant $c$. Moreover, given $\delta>0$ and $k=1,2, \ldots$,

$$
\left|\nabla^{k} u(x, t)\right| \leq c_{1}(k, \delta)
$$

for all $\left.(x, t) \in\left(\mathbb{R}_{+\delta}^{3} \backslash B^{+}\left(R_{1}\right)\right) \times\right]-5 / 4,0\left[\right.$. Here, $\mathbb{R}_{+\delta}^{3}:=\mathbb{R}_{+}^{3} \cap\left\{x_{3}>\delta\right\}$.

Proof By (4.8), we can state that

$$
\begin{aligned}
& \int_{-3 / 2}^{0} \int_{\mathbb{R}_{+}^{3} \backslash B^{+}(R)}|u|^{3} d x d t+\int_{-3 / 2}^{0}\left(\int_{\mathbb{R}_{+}^{3} \backslash B^{+}(R)}\left|\nabla p^{1}\right|^{\frac{9}{8}} d x\right)^{\frac{4}{3}} d t \\
& +\int_{-3 / 2}^{0}\left(\int_{\mathbb{R}_{+}^{3} \backslash B^{+}(R)}\left|\nabla p^{2}\right|^{\frac{10}{3}} d x\right)^{\frac{9}{20}} d t+\int_{-3 / 2}^{0}\left(\int_{\mathbb{R}_{+}^{3} \backslash B^{+}(R)}\left|\nabla p^{3}\right|^{2} d x\right)^{\frac{3}{4}} d t \rightarrow 0
\end{aligned}
$$

as $R \rightarrow \infty$. 
Given $\varepsilon>0$, there exists a positive number $R_{1}>0$ such that

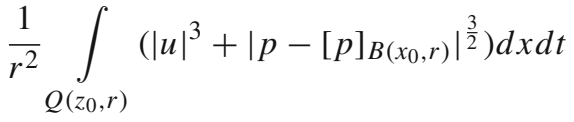

$$
\begin{aligned}
& \leq \frac{1}{r^{2}} \int_{Q\left(z_{0}, r\right)}|u|^{3} d x d t+\frac{c}{r^{2}} \sum_{i=1}^{3} \int_{Q\left(z_{0}, r\right)}\left|p^{i}-\left[p^{i}\right]_{B\left(x_{0}, r\right)}\right|^{\frac{3}{2}} d x d t \\
& \leq \frac{1}{r^{2}} \int_{Q\left(z_{0}, r\right)}|u|^{3} d x d t \\
& +c r^{-\frac{3}{2}} \int_{-3 / 2}^{0}\left(\int_{B\left(x_{0}, r\right)}\left|\nabla p^{1}\right|^{\frac{9}{8}} d x\right)^{\frac{4}{3}} d t+c r^{\frac{23}{20}} \int_{-3 / 2}^{0}\left(\int_{B\left(x_{0}, r\right)}\left|\nabla p^{2}\right|^{\frac{10}{3}} d x\right)^{\frac{9}{20}} d t \\
& +c r^{\frac{1}{4}} \int_{-3 / 2}^{0}\left(\int_{B\left(x_{0}, r\right)}\left|\nabla p^{3}\right|^{2} d x\right)^{\frac{3}{4}} d t<\varepsilon
\end{aligned}
$$

with $r=1 / 100$ and $\left.Q\left(z_{0}, r\right) \subset\left(\mathbb{R}_{+}^{3} \backslash B^{+}\left(R_{1} / 2\right)\right) \times\right]-3 / 2,0[$.

The same can be done for boundary points:

$$
\begin{aligned}
& \frac{1}{\rho^{2}} \int_{Q^{+}\left(z_{0}, \rho\right)}\left(|u|^{3}+\left|p-[p]_{B^{+}\left(x_{0}, \rho\right)}\right|^{\frac{3}{2}}\right) d x d t \leq \frac{1}{\rho^{2}} \int_{Q^{+}\left(z_{0}, \rho\right)}|u|^{3} d x d t \\
& +\frac{c}{\rho^{2}} \sum_{i=1}^{3} \int_{Q^{+}\left(z_{0}, \rho\right)}\left|p^{i}-\left[p^{i}\right]_{B^{+}\left(x_{0}, \rho\right)}\right|^{\frac{3}{2}} d x d t \leq \frac{1}{\rho^{2}} \int_{Q^{+}\left(z_{0}, \rho\right)}|u|^{3} d x d t \\
& +c \rho^{-\frac{3}{2}} \int_{-3 / 2}^{0}\left(\int_{B^{+}\left(x_{0}, \rho\right)}\left|\nabla p^{1}\right|^{\frac{9}{8}} d x\right)^{\frac{4}{3}} d t+c \rho^{\frac{23}{20}} \int_{-3 / 2}^{0}\left(\int_{B^{+}\left(x_{0}, \rho\right)}\left|\nabla p^{2}\right|^{\frac{10}{3}} d x\right)^{\frac{9}{20}} d t \\
& \quad+c \rho^{\frac{1}{4}} \int_{-3 / 2}^{0}\left(\int_{B^{+}\left(x_{0}, \rho\right)}\left|\nabla p^{3}\right|^{2} d x\right)^{\frac{3}{4}} d t<\varepsilon
\end{aligned}
$$

with $\varrho=1 / 10$ and $\left.Q^{+}\left(z_{0}, \varrho\right):=B^{+}\left(x_{0}, \varrho\right) \times\right] t_{0}-\varrho^{2}, t_{0}\left[\subset\left(\mathbb{R}_{+}^{3} \backslash B^{+}\left(R_{1} / 2\right)\right) \times\right]-$ $3 / 2,0\left[, x_{03}=0\right.$. From the $\varepsilon$-regularity theory developed in $[1,16,18]$, see details also in [3], in particular, we can show the validity of (4.15) in $\left.\left(\mathbb{R}_{+}^{3} \backslash B^{+}\left(3 R_{1} / 2\right)\right) \times\right]-$ 4/3, $0[$.

The second statement of the proposition can be deduced from the local regularity theory for the heat equation and bootstrap arguments applied to the vorticity equation and is described in detail in [23]. 


\section{Rescaling: scenario I}

Let us go back to our original problem (1.1)-(1.3).

We assume that $T>0$ is a blowup time. Theorem 1.1 can be proven ad absurdum. Suppose that there exists a sequence $t_{n} \uparrow T$ such that

$$
M:=\sup _{n}\left\|v\left(\cdot, t_{n}\right)\right\|_{L^{3, q}\left(\mathbb{R}_{+}^{3}\right)}<\infty
$$

then $T$ is NOT a blowup time.

It is known that there exists a global weak Leray-Hopf solution (energy solution) to initial boundary value problem (1.1)-(1.3). This solution coincides with $v$ on the interval ]0, $T$ [ and that is why we are going to denote it still by $v$. Arguments similar to used in the previous section show that for every $\epsilon>0$ there exists $R_{1}(\epsilon)>0$ such that

$$
\sup _{x \in \mathbb{R}_{+}^{3} \backslash B^{+}\left(R_{1}\right), \epsilon \leq t \leq T}|v(x, t)|<\infty .
$$

So, by the definition of blowup time $T$, there should be a singular point $x_{0}=$ $\left(x_{0}^{\prime}, x_{03}\right) \in \mathbb{R}_{+}^{3}$ at $t=T$, i.e., a point such that $\left.v \notin L_{\infty}\left(B\left(x_{0}, r\right) \cap \mathbb{R}_{+}^{3}\right) \times\right] T-r^{2}, T[)$ for any positive $r$. Without loss of generality, we may assume that $x_{0}^{\prime}=0$. Then one should consider two case

$$
x_{03}=0
$$

and

$$
x_{03}>0 \text {. }
$$

Now let us focus on the first case, which will be referred to as 'Scenario I'. The case $x_{03}>0$ will be referred to as 'Scenario II'. We know from $[16,18]$ that it must be

$$
\frac{1}{a^{2}} \int_{Q^{+}((0, T) ; a)}\left(|v|^{3}+\left|q-[q]_{B^{+}(a)}\right|^{\frac{3}{2}}\right) d x d t>\varepsilon
$$

for all $0<a<a_{0}$, for some positive $a_{0}$, and for some universal constant $\varepsilon$.

In this scenario, our rescaling will be as follows:

$$
u^{(n)}(y, s)=\lambda_{n} v(x, t), \quad p^{(n)}(y, s)=\lambda_{n}^{2} q(x, t),
$$

where

$$
x=\lambda_{n} y, \quad t=T+\lambda_{n}^{2} s, \quad \lambda_{n}=\sqrt{\frac{T-t_{n}}{2}}
$$


So, sufficiently smooth solutions $u^{(n)}$ and $p^{(n)}$ satisfy (4.1)-(4.3) with

$$
u_{0}^{(n)}(y)=\lambda_{n} v\left(\lambda_{n} y, t_{n}\right)
$$

Hence,

$$
\sup _{n}\left\|u_{0}^{(n)}\right\|_{L^{3, q}\left(\mathbb{R}_{+}^{3}\right)}=M .
$$

Without loss of generality, we may assume

$$
u_{0}^{(n)} \rightarrow u_{0}
$$

in $L^{3, q}\left(\mathbb{R}_{+}^{3}\right)$. So, all assumptions and statements of Propositions 4.1 and 4.2 hold for $u^{(n)}$ and $p^{(n)}$ and for their limits $u$ and $p$.

First examine the profile. Let us recall the known fact that $C_{0}^{\infty}\left(\mathbb{R}_{+}^{3}\right)$ is dense in $L^{s, p}\left(\mathbb{R}_{+}^{3}\right)$, for $1<s, p<\infty$. Also, recall that

$$
\left(L^{s, p}\left(\mathbb{R}_{+}^{3}\right)\right)^{\prime}=L^{s^{\prime}, p^{\prime}}\left(\mathbb{R}_{+}^{3}\right), \quad s^{\prime}=\frac{s}{s-1}, \quad p^{\prime}=\frac{p}{p-1} .
$$

The identification is as follows, if $f \in L^{s^{\prime}, p^{\prime}}\left(\mathbb{R}_{+}^{3}\right)$ and $g \in L^{s, p}\left(\mathbb{R}_{+}^{3}\right)$ :

$$
T_{f}(g)=\int_{\mathbb{R}_{+}^{3}} f g d x .
$$

Now, we mention that our assumption that $\left.v: \mathbb{R}_{+}^{3} \times\right] 0, \infty\left[\rightarrow \mathbb{R}^{3}\right.$ is a weak Leray Hopf solution implies that $v$ can be adjusted on some set of time of Lebesgue measure zero such that $v(\cdot, t) \in L_{2}\left(\mathbb{R}_{+}^{3}\right)$. Moreover, for any $\varphi(x) \in C_{0}^{\infty}\left(\mathbb{R}_{+}^{3}\right)$ the following functional is continuous in time on $[0, \infty[$ :

$$
t \rightarrow \int_{\mathbb{R}_{+}^{3}} v(x, t) \cdot \varphi(x) d x
$$

Our assumption that $\left\|v\left(\cdot, t_{n}\right)\right\|_{L^{3, q}\left(\mathbb{R}_{+}^{3}\right)} \leqslant M$ implies by O'Neil's inequality that for $\varphi \in C_{0}^{\infty}\left(\mathbb{R}^{3}\right)$ :

$$
\left|\int_{\mathbb{R}_{+}^{3}} v\left(x, t_{n}\right) \cdot \varphi(x) d x\right| \leqslant M\|\varphi\|_{L^{\frac{3}{2}, q^{\prime}}\left(\mathbb{R}_{+}^{3}\right)} .
$$


Hence, the weak-continuity implies

$$
\left|\int_{\mathbb{R}_{+}^{3}} v(x, T) \cdot \varphi(x) d x\right| \leqslant M\|\varphi\|_{L^{\frac{3}{2}, q^{\prime}}\left(\mathbb{R}_{+}^{3}\right)} .
$$

This implies, from the aforementioned facts regarding Lorentz spaces, that

$$
\|v(\cdot, T)\|_{L^{3, q}\left(\mathbb{R}_{+}^{3}\right)} \leqslant M
$$

Now, we shall show that

$$
u(x, 0)=0
$$

Indeed, it follows from (4.12)-(4.14) that

$$
\int_{B^{+}(a)}\left|u^{(n)}(x, 0)\right| d x \rightarrow \int_{B^{+}(a)}|u(x, 0)| d x
$$

Using generalized Holder inequality for Lorentz spaces (along with scale invariance), it is not so difficult to show

$$
\frac{1}{a^{2}} \int_{B^{+}(a)}\left|u^{(n)}(x, 0)\right| d x \leqslant c\left\|u^{(n)}(\cdot, 0)\right\|_{L^{3, q}\left(B^{+}(a)\right)}=c\|v(\cdot, T)\|_{L^{3, q}\left(B^{+}\left(\lambda_{n} a\right)\right)} .
$$

Now $v(\cdot, T) \in L_{3, q}\left(\mathbb{R}_{+}^{3}\right)$ and obviously $d_{\left.v(\cdot, T), B^{+}\left(\lambda_{n} a\right)\right)}(\alpha) \rightarrow 0$. This implies,

$$
\|v(\cdot, T)\|_{L^{3, q}\left(B^{+}\left(\lambda_{n} a\right)\right)} \rightarrow 0 .
$$

Now, we need to show that the limit function is not identically zero. Fix $0<a_{*}<$ $1 / 4$ then we have

$$
\begin{aligned}
& \frac{1}{a^{2}} \int_{Q^{+}(a)}\left(\left|u^{(n)}\right|^{3}+\left|p^{(n)}-\left[p^{(n)}\right]_{B^{+}(a)}\right|^{\frac{3}{2}}\right) d x d t \\
& =\frac{1}{\left(\lambda_{n} a^{2}\right)} \int_{Q^{+}\left((0, T), \lambda_{n} a\right)}\left(|v|^{3}+\left|q-[q]_{B^{+}\left(\lambda_{n} a\right)}\right|^{\frac{3}{2}}\right) d x d t>\varepsilon
\end{aligned}
$$

for $0<a<a_{*}$. 
We know that

$$
\begin{aligned}
M_{1}\left(a_{*}\right):= & \sup _{n}\left\{\frac{1}{\left(2 a_{*}\right)^{2}} \int_{Q^{+}\left(2 a_{*}\right)}\left(\left|u^{(n)}\right|^{3}+\left|p^{(n)}-\left[p^{(n)}\right]_{B^{+}\left(2 a_{*}\right)}\right|^{\frac{3}{2}}\right) d x d t\right. \\
& \left.+\sup _{-\left(2 a_{*}\right)^{2}<t<0}\left\|u^{(n)}(\cdot, t)\right\|_{2, B^{+}\left(2 a_{*}\right)}^{2}+\left\|\nabla u^{(n)}\right\|_{2, Q^{+}\left(2 a_{*}\right)}^{2}\right\}<\infty
\end{aligned}
$$

Let us fix a $C^{2}$-domain $\Omega_{*}$ such that $B^{+}\left(a_{*}\right) \subset \Omega_{*} \subset B^{+}\left(2 a_{*}\right)$ and let $Q_{*}=$ $\left.\Omega_{*} \times\right]-a_{*}^{2}, 0[$. We may use the same type of decompositions as in the previous sections

$$
u^{(n)}=w^{1}+w^{2}, \quad p^{(n)}=r^{1}+r^{2}
$$

so that

$$
\partial_{t} w^{1}-\Delta w^{1}+\nabla r^{1}=0, \quad \operatorname{div} w^{1}=0
$$

in $Q_{*}$,

$$
w^{1}\left(x^{\prime}, 0, t\right)=0
$$

for all $(x, t) \in \partial \Omega_{*} \times\left[-a_{*}^{2}, 0\right]$ with $x_{3}=0$,

$$
w^{1}\left(x,-a_{*}^{2}\right)=u^{(n)}\left(x,-a_{*}^{2}\right)
$$

for all $x \in \Omega_{*}$ and

$$
\partial_{t} w^{2}-\Delta w^{2}+\nabla r^{2}=-\operatorname{div} u^{(n)} \otimes u^{(n)}, \quad \operatorname{div} w^{2}=0
$$

in $Q_{*}$,

$$
w^{2}=0
$$

on the parabolic boundary of $Q_{*}$.

By coercive estimate for the linear Stokes system with different time and space exponents, see $[6,24]$, we have

$$
\left\|w^{2}\right\|_{W_{\frac{12}{11}, \frac{3}{2}}^{2,1}\left(Q_{*}\right)}+\left\|r^{2}\right\|_{W_{\frac{12}{11}, \frac{3}{2}}^{1,0}\left(Q_{*}\right)} \leqslant C\left(a_{*}\right)\left\|u^{(n)} \cdot \nabla u^{(n)}\right\|_{L_{\frac{12}{11}, \frac{3}{2}}\left(Q_{*}\right)} .
$$

Using (5.4) one infers

$$
\int_{Q^{+}\left(a_{*}\right)}\left|r^{2}-\left[r^{2}\right]_{B^{+}\left(a_{*}\right)}\right|^{\frac{3}{2}} d x d t \leq c\left(a_{*}\right) \int_{-a_{*}^{2}}^{0}\left(\int_{B^{+}\left(a_{*}\right)}\left|\nabla r^{2}\right|^{\frac{12}{11}} d x\right)^{\frac{11}{8}} d t
$$




$$
\begin{aligned}
& \leq c\left(a_{*}\right) \int_{-a_{*}^{2}}^{0}\left(\int_{\Omega_{*}}\left|\nabla r^{2}\right|^{\frac{12}{11}} d x\right)^{\frac{11}{8}} d t \\
& \leq c\left(a_{*}\right) \int_{-a_{*}^{2}}^{0}\left(\int_{\Omega_{*}}\left|u^{(n)} \cdot \nabla u^{(n)}\right|^{\frac{12}{11}} d x\right)^{\frac{11}{8}} d t \\
& \leq c\left(a_{*}\right)\left\|\nabla u^{(n)}\right\|_{L_{2}\left(Q_{*}\right)}^{\frac{3}{2}}\left\|u^{(n)}\right\|_{L_{2, \infty}\left(Q_{*}\right)}^{\frac{3}{4}}\left\|u^{(n)}\right\|_{L_{3}\left(Q_{*}\right)}^{\frac{3}{4}} \\
& \leq c\left(a_{*}, M_{1}\right)\left\|u^{(n)}\right\|_{L_{3}\left(Q_{*}\right)}^{\frac{3}{4}} \leq c\left(a_{*}, M_{1}\right)\left(\int_{Q^{+}\left(2 a_{*}\right)}\left|u^{(n)}\right|^{3} d x d t\right)^{\frac{1}{4}} .
\end{aligned}
$$

By the local regularity theory up to the boundary for the Stokes system developed in $[16,18]$ and by $(5.3)-(5.5)$, for any $s>\frac{12}{11}$,

$$
\begin{aligned}
& \int_{-\left(a_{*} / 2\right)^{2}}\left(\int_{B^{+}\left(a_{*} / 2\right)}\left|\nabla r^{1}\right|^{s} d x\right)^{\frac{3}{2 s}} d t \\
& \leq c\left(a_{*}, s\right)\left(\left\|w^{1}\right\|_{L_{\frac{12}{11}, \frac{3}{2}}^{\frac{3}{2}}\left(Q^{+}\left(a_{*}\right)\right)}+\left\|\nabla w^{1}\right\|_{L_{\frac{12}{11}, \frac{3}{2}}^{\frac{3}{2}}\left(Q^{+}\left(a_{*}\right)\right)}^{\frac{3}{2}}\right) \\
& \left.\quad+\left\|r^{1}-\left[r^{1}\right]_{B^{*}\left(a_{*}\right)}\right\|_{L_{\frac{12}{11}, \frac{3}{2}}\left(Q^{+}\left(a_{*}\right)\right)}\right) \\
& \leq c\left(a_{*}, s\right)\left(\left\|w^{2}\right\|_{L_{\frac{12}{11}, \frac{3}{2}}^{\frac{3}{2}}\left(Q^{+}\left(a_{*}\right)\right)}+\left\|\nabla w^{2}\right\|_{L_{\frac{12}{11}}, \frac{3}{2}}^{\frac{3}{2}}\left(Q^{+}\left(a_{*}\right)\right)\right. \\
& \quad+\left\|u^{n}\right\|_{L_{\frac{12}{11}, \frac{3}{2}}^{\frac{3}{2}}\left(Q^{+}\left(a_{*}\right)\right)}+\left\|\nabla u^{n}\right\|_{L_{\frac{12}{11}, \frac{3}{2}}^{\frac{3}{2}}\left(Q^{+}\left(a_{*}\right)\right)} \\
& \left.\quad+\int_{Q^{+}\left(a_{*}\right)}\left|r^{1}-\left[r^{1}\right]_{B^{*}\left(a_{*}\right)}\right|^{\frac{3}{2}} d x d t\right) \\
& \leq c\left(a_{*}, M_{1}, s\right)\left(\int_{Q^{+}\left(a_{*}\right)}\left(\left|p^{(n)}-\left[p^{(n)}\right]_{B^{+}\left(a_{*}\right)}\right|^{\frac{3}{2}}+\left|r^{2}-\left[r^{2}\right]_{B^{*}\left(a_{*}\right)}\right|^{\frac{3}{2}}\right) d x d t+1\right) \\
& \leq c\left(a_{*}, M_{1}\right) .
\end{aligned}
$$

Next, we have for $0<a<a_{*} / 2$ and for $s=9$,

$$
\begin{aligned}
\varepsilon & <\frac{1}{a^{2}} \int_{Q^{+}(a)}\left(\left|p^{(n)}-\left[p^{(n)}\right]_{B^{+}(a)}\right|^{\frac{3}{2}}+\left|u^{(n)}\right|^{3}\right) d x d t \\
& \leq \frac{1}{a^{2}} \int_{Q^{+}(a)}\left(\left|r^{1}-\left[r^{1}\right]_{B^{+}(a)}\right|^{\frac{3}{2}}\right) d x d t
\end{aligned}
$$




$$
\begin{aligned}
& +\frac{1}{a^{2}} \int_{Q^{+}(a)}\left(\left|r^{2}-\left[r^{2}\right]_{B^{+}(a)}\right|^{\frac{3}{2}}+\left|u^{(n)}\right|^{3}\right) d x d t \\
\leq & c\left(a_{*}, M_{1}\right) a^{2}+\frac{1}{a^{2}} \int_{Q^{+}(a)}\left(\left|r^{2}-\left[r^{2}\right]_{B^{+}(a)}\right|^{\frac{3}{2}}+\left|u^{(n)}\right|^{3}\right) d x d t \\
\leq & \frac{c\left(a_{*}\right)}{a^{2}} \int_{Q^{+}\left(a_{*}\right)}\left(\left|u^{(n)}\right|^{3}+\left|r^{2}-\left[r^{2}\right]_{B^{+}\left(a_{*}\right)}\right|^{\frac{3}{2}}\right) d x d t+c\left(a_{*}, M_{1}\right) a^{2} \\
\leq & \frac{c\left(a_{*}\right)}{a^{2}} \int_{Q^{+}\left(a_{*}\right)}\left|u^{(n)}\right|^{3} d x d t+\frac{c\left(a_{*}, M_{1}\right)}{a^{2}}\left(\int_{Q^{+}\left(2 a_{*}\right)}\left|u^{(n)}\right|^{3} d x d t\right)^{\frac{1}{4}}+c\left(a_{*}, M_{1}\right) a^{2} \\
\leq & \frac{c\left(a_{*}, M_{1}\right)}{a^{2}}\left(\int_{Q^{+}\left(2 a_{*}\right)}\left|u^{(n)}\right|^{3} d x d t\right)^{\frac{1}{4}}+c\left(a_{*}, M_{1}\right) a^{2} .
\end{aligned}
$$

For sufficiently small $a>0$,

$$
0<\varepsilon / 2<\varepsilon-c\left(a_{*}, M_{1}\right) a^{2} \leq \frac{c\left(a_{*}, M_{1}\right)}{a^{2}}\left(\int_{Q^{+}\left(2 a_{*}\right)}\left|u^{(n)}\right|^{3} d x d t\right)^{\frac{1}{4}}
$$

and thus

$$
\int_{Q^{+}\left(2 a_{*}\right)}\left|u^{(n)}\right|^{3} d x d t>\left(\frac{a^{2} \varepsilon}{c\left(a_{*}, M_{1}\right)}\right)^{4} .
$$

Passing to the limit as $n \rightarrow \infty$, we find

$$
\int_{Q^{+}\left(2 a_{*}\right)}|u|^{3} d x d t>\left(\frac{a^{2} \varepsilon}{c\left(a_{*}, M_{1}\right)}\right)^{4} .
$$

Next, we follow arguments of the paper [3] that related with backward uniqueness for the heat operator with lower order terms. By Proposition 4.2, we have

$$
\left|\partial_{t} \omega-\Delta \omega\right| \leq c(|\omega|+|\nabla \omega|),|\omega| \leqslant c
$$

in $\left.\left\{x \in \mathbb{R}^{3}: x_{3}>2 R_{1}\right\} \times\right]-5 / 4,0[$, where $\omega=\nabla \wedge u$. Next recall (5.2), which implies

$$
\omega(x, 0)=0
$$


for $x \in\left\{\mathbb{R}^{3}: x_{3}>2 R_{1}\right\}$. Thus, we can apply arguments from the paper [3] that are related with backward uniqueness for the heat operator with lower order terms (Theorem 5.1 of [3]) to obtain

$$
\omega(x, t)=0
$$

for all $\left.(x, t) \in\left\{x \in \mathbb{R}^{3}: x_{3}>2 R_{1}\right\} \times\right]-6 / 5,0$ [. Recall that from Proposition 4.2 that for arbitrary $\delta>0$ and $k=0,1,2, \ldots$ we have the decay estimate for spatial derivatives,

$$
\left|\nabla^{k} u(x, t)\right| \leq c_{1}(k, \delta)
$$

valid for all $\left.(x, t) \in\left(\mathbb{R}_{+\delta}^{3} \backslash B^{+}\left(R_{1}\right)\right) \times\right]-5 / 4,0[$. This allows one to apply unique continuation through spatial boundaries (Theorem 3.1 of [3]) to conclude that (5.7) is valid in $\left.\left(\mathbb{R}_{+}^{3} \backslash B^{+}\left(R_{1}\right)\right) \times\right]-7 / 6,0[$.

Since $\operatorname{div} u(\cdot, t)=0$ in $\mathbb{R}_{+}^{3} \backslash B^{+}\left(R_{1}\right)$ with $u\left(x^{\prime}, 0, t\right)=0$ for $\mathrm{t}$ in ] $-\frac{6}{5}$, 0 [, we see that for $u=\left(u_{i}(x, t)\right)_{i=1,2,3}$ we have

$$
\frac{\partial u_{3}\left(x^{\prime}, 0, t\right)}{\partial x_{3}}=-\frac{\partial u_{1}\left(x^{\prime}, 0, t\right)}{\partial x_{1}}-\frac{\partial u_{2}\left(x^{\prime}, 0, t\right)}{\partial x_{2}}=0
$$

for $x=\left(x^{\prime}, 0\right) \in \mathbb{R}_{+}^{3} \backslash B^{+}\left(R_{1}\right)$ and a.a $t$ in $]-\frac{6}{5}, 0[$. Additionally, since $\nabla \times u(\cdot, t)=0$ in $\mathbb{R}_{+}^{3} \backslash B^{+}\left(R_{1}\right)$ with $u\left(x^{\prime}, 0, t\right)=0$ for $\mathrm{t}$ in $]-\frac{6}{5}, 0[$, we see that we have

$$
\frac{\partial u_{1}\left(x^{\prime}, 0, t\right)}{\partial x_{3}}=\frac{\partial u_{3}\left(x^{\prime}, 0, t\right)}{\partial x_{1}}=0
$$

and

$$
\frac{\partial u_{2}\left(x^{\prime}, 0, t\right)}{\partial x_{3}}=\frac{\partial u_{3}\left(x^{\prime}, 0, t\right)}{\partial x_{2}}=0
$$

for $x=\left(x^{\prime}, 0\right) \in \mathbb{R}_{+}^{3} \backslash B^{+}\left(R_{1}\right)$ and the same $t$. From (5.9)-(5.11), one can deduce that

$$
\nabla u\left(x^{\prime}, 0, t\right)=0
$$

for $x=\left(x^{\prime}, 0\right) \in \mathbb{R}_{+}^{3} \backslash B^{+}\left(R_{1}\right)$ and the same $t$. Indeed, one may argue further to conclude that for any multi-index $\alpha$

$$
D^{\alpha} u\left(x^{\prime}, 0, t\right)=0
$$

for $x=\left(x^{\prime}, 0\right) \in \mathbb{R}_{+}^{3} \backslash B^{+}\left(R_{1}\right)$ and a.a $t$ in $]-\frac{6}{5}, 0[$. Using $\Delta u(\cdot, t)=0$ in $\mathbb{R}_{+}^{3} \backslash B^{+}\left(R_{1}\right)$, one can also infer that an appropriate extension of $u(\cdot, t)$ is harmonic 
and hence analytic in $\mathbb{R}^{3} \backslash B\left(R_{1}\right)$. From the analyticity of this extension and (5.13), one can deduce that

$$
u(x, t)=0
$$

for all $x \in \mathbb{R}_{+}^{3} \backslash B^{+}\left(2 R_{1}\right)$ and for the same $t$. Recall, from (4.12)-(4.14) that we have continuity on $\left[-\frac{6}{5}, 0\right]$ for the following functional in time (here $\varphi \in C_{0}^{\infty}\left(\mathbb{R}^{3}\right)$ ):

$$
t \rightarrow \int_{\mathbb{R}_{+}^{3}} \varphi(x) \cdot u(x, t) d x
$$

Now, (5.14) also implies that there exists $\Sigma \subset]-\frac{6}{5}, 0[$ with $|\Sigma|=0$ such that for $t \in]-\frac{6}{5}, 0[\backslash \Sigma$ :

$$
\int_{\mathbb{R}_{+}^{3}}|\nabla u|^{2} d x<\infty .
$$

Recall from Proposition 4.1 that $(u, p)$ satisfy a local energy inequality. These facts imply that there exists a sufficiently smooth bounded domain $\Omega \subset \mathbb{R}_{+}^{3}$ and a set $\left.\Sigma^{\prime} \subset \Sigma \subset\right]-\frac{6}{5}, 0\left[\right.$ such that for any $t_{0} \in \Sigma^{\prime}, u$ is a Leray-Hopf solution to (1.1)(1.3) on $\Omega \times] t_{0}, 0$ [ with initial data that additionally has finite dirichlet norm (5.16) over $\Omega$. By local in time solvability for the Navier-Stokes equations with initial data with finite Dirichlet integral, see details in [3,21], one can infer that there exists $\delta_{0}>0$ such that for any $0<\epsilon<\delta_{0}$ and $k=1,2 \ldots$ :

$$
\sup _{t_{0}+\epsilon<t<t_{0}+\delta-\epsilon} \sup _{\Omega}\left|\nabla^{k} u(x, t)\right|<\infty .
$$

An application of unique continuation through spatial boundaries allows us to deduce that $\omega=0$ in $\left.\mathbb{R}_{+}^{3} \times\right] t_{0}+\epsilon, t_{0}+\delta-\epsilon$. Using this, along with $u \in L_{\frac{10}{3}}\left(Q_{-\frac{6}{5}, 0}^{+}\right)$and (5.15), gives that $u=0$ in $\left.\mathbb{R}_{+}^{3} \times\right]-\frac{6}{5}, 0[$.

The latter contradicts with (5.6) and thus $T$ is not a blowup time.

\section{Rescaling: scenario II}

Recall that 'Scenario II' refers to the case $x_{03}>0$ for the singular point at blowup time $T$. Here, the scaling is $x=x_{0}+\lambda_{n} y$. So, we replace $\mathbb{R}_{+}^{3}$ with $\mathbb{R}_{h}^{3}=\left\{y=\left(y^{\prime}, y_{3}\right) \in\right.$ $\left.\mathbb{R}: y_{3}>h\right\}$ with $h=h_{n}=-x_{03} / \lambda_{n}$.

In the case, sufficiently smooth functions $u^{(n)}$ and $p^{(n)}$ are a solution to the following initial boundary value problem:

$$
\partial_{t} u^{(n)}+\operatorname{div}^{(n)} \otimes u^{(n)}-\Delta u^{(n)}=-\nabla p^{(n)}, \quad \operatorname{div} u=0
$$


in $\left.\mathbb{R}_{h_{n}}^{3} \times\right]-2,0[$,

$$
u^{(n)}\left(x^{\prime},-h_{n}, t\right)=0
$$

for $\left(x^{\prime}, t\right) \in \mathbb{R}^{2} \times[-2,0]$,

$$
u^{(n)}(\cdot,-2)=u_{0}^{(n)}(\cdot) \in L^{3, q}\left(\mathbb{R}_{h_{n}}^{3}\right)
$$

and

$$
\sup _{n}\left\|u_{0}^{(n)}\right\|_{L^{3, q}\left(\mathbb{R}_{h_{n}}^{3}\right)} \leq M .
$$

Without loss of generality, we may assume

$$
u_{0}^{(n)} \rightarrow u_{0}
$$

in $L^{3, q}\left(\mathbb{R}_{h}^{3}\right)$ for any $h>-\infty$.

We can use estimates of Sect. 2 in domains $\mathbb{R}_{h_{n}}^{3}$ with constants independent of $n$.

Proposition 6.1 There exist subsequences still denoted in the same way with the following properties:

$$
u^{(n)} \rightarrow u
$$

in $L_{\frac{10}{3}}\left(\mathbb{R}_{h}^{3} \times\right]-2,0[)$ for any $h>-\infty$ with $u \in L_{\frac{10}{3}}\left(Q_{-2,0}\right)$ and $\left.Q_{-2,0}=\mathbb{R}^{3} \times\right]-$ $2,0[$,

$$
\nabla u^{(n)} \rightarrow \nabla u
$$

in $L_{2}(B(R) \times]-2+\delta, 0[)$ for any $R>0$ and any $0<\delta<2$,

$$
u^{(n)} \rightarrow u
$$

in $L_{3}(B(R) \times]-3 / 2$, 0[) for any $R>0$;

$$
p^{(n)} \rightarrow p
$$

in $L_{\frac{3}{2}}(B(R) \times]-3 / 2,0[)$ for any $R>0$.

Functions $u$ and $p$ satisfy the Navier-Stokes system in $\mathbb{Q}_{-3 / 2,0}$.

For the pressure $p$, the following global estimates are valid:

$$
p=\sum_{i=1}^{3} p^{i}
$$


with the estimates

$$
\left\|\nabla p^{1}\right\|_{L_{\frac{9}{8}, \frac{3}{2}}\left(Q_{-3 / 2,0}\right)}+\left\|\nabla p^{2}\right\|_{L_{\frac{10}{3}}\left(Q_{-3 / 2,0}\right)}++\left\|\nabla p^{3}\right\|_{L_{2}\left(Q_{-3 / 2,0}\right)}<\infty
$$

Moreover, for any $R>0$, the limits pair $u$ and $p$ satisfies the local energy inequality

$$
\begin{aligned}
& \int_{B\left(x_{0}, R\right)} \varphi^{2}(x, t)|u(x, t)|^{2} d x+2 \int_{t_{0}-R^{2}}^{t} \int_{B\left(x_{0}, R\right)} \varphi^{2}|\nabla u|^{2} d x d t \\
& \leq \int_{t_{0}-R^{2}}^{t} \int_{\left.B\left(x_{0}, R\right)\right)}|u|^{2}\left(\partial_{t} \varphi^{2}+\Delta \varphi^{2}\right)+u \cdot \nabla \varphi^{2}\left(|u|^{2}+2 p\right) d x d s
\end{aligned}
$$

for all $-3 / 2<t_{0}-R^{2}<t \leq t_{0} \leq 0$, for all $x_{0} \in \mathbb{R}^{3}$, and for all $\varphi \in$ $C_{0}^{\infty}\left(B\left(x_{0}, R\right) \times\right] t_{0}-R^{2}, t_{0}+R^{2}[)$.

The proof of Proposition 6.1 goes along the lines of the proof of Proposition 4.1 with minor modifications. Scenario II can then be ruled out in the same way as in [21].

We would like to stress that a major simplification in Scenario II, compared with Scenario I, is related to showing non-triviality of the limit solution. In the interior case we may follow the local pressure decomposition used in [21] one of which is harmonic and the other satisfies a coercive estimate. Interior properties of harmonic functions are essential in the use of this decomposition for showing non-triviality of the limit function. In the boundary case of Scenario I the same decomposition doesn't apply. Instead, in order to show non-triviality, one uses a local decomposition of the velocity and pressure together with estimates for the Stokes system near the boundary as described in Scenario I.

Acknowledgements The authors would like to take the opportunity to thank the anonymous referees for their invaluable comments that helped improve the presentation of the paper.

Open Access This article is distributed under the terms of the Creative Commons Attribution 4.0 International License (http://creativecommons.org/licenses/by/4.0/), which permits unrestricted use, distribution, and reproduction in any medium, provided you give appropriate credit to the original author(s) and the source, provide a link to the Creative Commons license, and indicate if changes were made.

\section{References}

1. Caffarelli, L., Kohn, R.-V., Nirenberg, L.: Partial regularity of suitable weak solutions of the NavierStokes equations. Commun. Pure Appl. Math. XXXV, 771-831 (1982)

2. Calderon, C.P.: Existence of weak solutions for the Navier-Stokes equations with initial data in Lp. Trans. Am. Math. Soc. 318(1), 179-200 (1990)

3. Escauriaza, L., Seregin, G., Šverák, V.: $L_{3, \infty}$-solutions of Navier-Stokes equations and backward uniqueness. (Russian) Uspekhi Mat. Nauk 58(2)(350), 3-44 (2003) [translation in. Russ. Math. Surv. 58(2), 211-250 (2003)]

4. Koch, G., Gallagher, I., Planchon, F.: Blow-up of critical Besov norms at a potential Navier-Stokes singularity. Commun. Math. Phys. ISSN 0010-3616 (2016) 
5. Giga, Y.: Solutions for semilinear parabolic equations in $L_{p}$ and regularity of weak solutions of the Navier- Stokes system. J. Differ. Equ. 62, 186-212 (1986)

6. Giga, Y., Sohr, H.: Abstract $L_{p}$ estimates for the Cauchy problem with applications to the Navier-Stokes equations in exterior domains. J. Funct. Anal. 102(1), 72-94 (1991)

7. Kikuchi, N., Seregin, G.: Weak solutions to the Cauchy problem for the Navier-Stokes equations satisfying the local energy inequality. AMS Transl. Ser. 2(220), 141-164 (2007)

8. Kim, Hyunseok: Kozono, H.: Interior regularity criteria in weak spaces for the Navier-Stokes equations. Manuscr. Math. 115(1), 85-100 (2004)

9. Kozono, H.: Removable singularities of weak solutions to the Navier-Stokes equations. Commun. Partial Differ. Equ. 23(5-6), 949-966 (1998)

10. Lemarie-Rieusset, P.G.: Recent developments in the Navier-Stokes problem, Reseacrh notes in mathematics series, vol. 431. Chapman \& Hall/CRC, Boca Raton, FL (2002)

11. Leray, J.: Sur le mouvement d'un liquide visqueux emplissant l'espace. Acta Math. 63, 193-248 (1934)

12. Luo, Y., Tsai, T.P.: Regularity criteria in weak $L_{3}$ for $3 \mathrm{D}$ incompressible Navier-Stokes equations (2014). arXiv: 1310.8307

13. McCormick, D.S., Robinson, J.C., Rodrigo, J.L.: Generalised Gagliardo-Nirenberg inequalities using weak Lebesgue spaces and BMO. Milan J. Math. 81(2), 265-289 (2013)

14. Mikhailov, A.S., Shilkin, T.N.: L 3, $\infty$-solutions to the 3D-Navier-Stokes system in the domain with a curved boundary. Zap. Nauchn. Sem. S.-Peterburg. Otdel. Mat. Inst. Steklov. (POMI) 336 (2006), Kraev. Zadachi Mat. Fiz. i Smezh. Vopr. Teor. Funkts. 37, 133-152, 276 [translation in J. Math. Sci. (N. Y.) 143(2), 2924-2935 (2007)]

15. Phuc, N.C.: The Navier-Stokes equations in nonendpoint borderline Lorentz spaces (2014). arXiv: 1407.5129

16. Seregin, G.A.: Local regularity of suitable weak solutions to the Navier-Stokes equations near the boundary. J. Math. Fluid Mech. 4(1), 1-29 (2002)

17. Seregin, G.A.: On smoothness of $L_{3, \infty}$-solutions to the Navier-Stokes equations up to boundary. Mathematische Annalen 332, 219-238 (2005)

18. Seregin, G.: A note on local boundary regularity for the Stokes system. Zapiski Nauchn. Seminar. POMI 370, 151-159 (2009)

19. Seregin, G.: A note on necessary conditions for blow-up of energy solutions to the Navier-Stokes equations, Progress in Nonlinear Differential Equations and Their Applications, vol. 60, pp. 631-645. Springer, Basel AG (2011)

20. Seregin, G.: Necessary conditions of potential blow up for the Navier-Stokes equations. Zapiski Nauchn. Seminar. POMI 385, 187-199 (2010)

21. Seregin, G.: A certain necessary condition of potential blow up for Navier-Stokes equations. Commun. Math. Phys. 312(3), 833-845 (2012)

22. Seregin, G., Šverák, V.: On global weak solutions to the Cauchy problem for the Navier-Stokes equations with large $L_{3}$ initial data. Nonlinear Anal. (2016). doi:10.1016/j.na.2016.01.018

23. Serrin, J.: On the interior regularity of weak solutions of the Navier-Stokes equations. Arch. Ration. Mech. Anal. 9, 187-195 (1962)

24. Sohr, H., von Wahl, W.: On the regularity of the pressure of weak solutions of Navier-Stokes equations. Arch. Math. (Basel) 46(5), 428-439 (1986)

25. Solonnikov, V.A.: Estimates of solutions to the non-stationary Navier-Stokes system. Zapiski Nauchn. Seminar. LOMI 28, 153-231 (1973)

26. Solonnikov, V.A.: Estimates for solutions of the nonstationary Stokes problem in anisotropic Sobolev spaces and estimates for the resolvent of the Stokes operator (Russian). Uspekhi Mat. Nauk 58(2)(350), 123-156 (2003) [translation in. Russ. Math. Surv. 58(2), 331-365 (2003)]

27. Ukai, S.: A solution formula for the Stokes equation in $\mathbb{R}_{+}^{n}$. Commun. Pure Appl. Math. 40(5), 611-621 (1987)

28. Wang, W., Zhang, Z.: On the interior regularity criterion and the number of singular points to the Navier-Stokes equations (2012). arXiv:1201.1100 